\title{
ON THE REALIZATION AND CLASSIFICATION OF SYMMETRIC ALGEBRAS AS COHOMOLOGY RINGS
}

\author{
LARRY SMITH
}

ABSTRACT. In this note, we prove that every algebra of the form $E\left[\sigma, \ldots, \sigma_{n}\right] \otimes$ $P\left[\rho_{1}, \ldots, \rho_{n}\right]$ that is an unstable algebra over the $\bmod p$ Steenrod algebra, $p$ an odd prime, that satisfies

$$
\beta \sigma_{i}=\rho_{i}, \quad \operatorname{deg} \rho_{i} \neq 0(p), \quad i=1, \ldots, n,
$$

arises as the cohomology of at least one topological space. Moreover, we show that the classification of such algebras is implicit in the work of Adams and Wilkerson and Clark and Ewing.

Let $p$ be a prime integer. The question of which graded polynomial algebras can be realized by the $\mathbf{Z} / p$ cohomology of a topological space has been solved in the nonmodular case (i.e. the case where none of the polynomial generators has a degree divisible by $p$ ) by the construction of Clark and Ewing [6] and the classification of Adams and Wilkerson [1]. Taken together these results may be interpreted as follows. Suppose, given an unstable polynomial algebra over the mod $p$ Steenrod algebra (see $[1,14]$ for terminology) $P\left[\rho_{1}, \ldots, \rho_{n}\right]$, where $p \nmid \operatorname{deg} \rho_{i}, i=1, \ldots, n$. Then there exists a finite $p$-adic generalized reflection group $G<\mathrm{GL}\left(n ; \mathbf{Z}_{p}\right)$ of order prime to $p$ such that

$$
P\left[\rho_{1}, \ldots, \rho_{n}\right] \simeq P(\bar{V})^{G}
$$

where $\bar{V}:=\mathbf{Z} / p \otimes_{\mathbf{Z}_{p}}\left(\mathbf{Z}_{p}\right)^{n}$, the action of $G$ being via reduction $G \rightarrow \operatorname{GL}\left(n ; \mathbf{Z}_{p}\right) \rightarrow$ $\mathrm{GL}(n, \mathrm{Z} / p)$, and there is a topological space $X(V, G)$ such that

$$
H^{*}(X(V ; G) ; \mathbf{Z} / p) \simeq P\left[\rho_{1}, \ldots, \rho_{n}\right]
$$

as algebras over the Steenrod algebra, where $V:=\left(\mathbf{Z}_{p}\right)^{n}$.

In this note we consider the analogous situation for unstable symmetric algebras over the Steenrod algebra of the form

$$
E\left(\sigma_{1}, \ldots, \sigma_{n}\right) \otimes P\left[\rho_{1}, \ldots, \rho_{n}\right],
$$

where $p$ is an odd prime, and the $\bmod p$ Bockstein acts via $\beta \sigma_{i}=\rho_{i} ; i=1, \ldots, n$, and the nonmodular condition holds:

$$
\operatorname{deg} \rho_{i} \neq 0(p), \quad i=1, \ldots, n
$$

Received by the editors February 11, 1982.

1980 Mathematics Subject Classification. Primary 55R99, 55R40, 55P45.

(c) 1983 American Mathematical Society $0002-9939 / 82 / 0000-0888 / \$ 02.25$ 
For such algebras we provide the complete analog of the Clark and Ewing and Adams and Wilkerson results. Specifically we will prove

THEOREM. Let $p$ be an odd prime and

$$
S^{*}=E\left[\sigma_{1}, \ldots, \sigma_{n}\right] \otimes P\left[\rho_{1}, \ldots, \rho_{n}\right]
$$

an unstable algebra over the Steenrod algebra, where

$$
\left.\begin{array}{l}
\beta \sigma_{i}=\rho_{i} \\
\operatorname{deg} \rho_{i} \geq 0 \bmod p
\end{array}\right\}, \quad i=1, \ldots, n .
$$

Then there exists a finite p-adic hyperplane group $G<\mathrm{GL}\left(n ; \mathbf{Z}_{p}\right)$ of order prime to $p$ such that

$$
P\left[\rho_{1}, \ldots, \rho_{n}\right] \simeq P(\bar{V})^{G}
$$

where $\bar{V}:=\mathbf{Z} / p \otimes_{\mathbf{Z}_{p}}\left(\mathbf{Z}_{p}\right)^{n}$. Moreover there is a topological space $Y(V ; G)$ such that

$$
H^{*}(Y(V, G) ; \mathrm{Z} / p) \simeq S^{*}
$$

as algebras over the Steenrod algebra.

This generalizes $[2, \S 2]$ for the case $n=1$. For the proof we require a number of preliminary maneuvers. We begin with the construction of the space $Y(V, G)$ associated to a finite $p$-adic hyperplane group $G<\mathrm{GL}\left(V ; \mathbf{Z}_{p}\right)$.

$N$.B. Given a mod $p$ hyperplane group $G<\mathrm{GL}(\bar{V} ; \mathbf{Z} / p)$ one can induce a free action of $G$ on the Eilenberg-Mac Lane space $K(\bar{V}, 1)$; here $\bar{V}:=(\mathbf{Z} / p)^{n}$. Since

$$
H^{*}(K(\bar{V}, 1) ; \mathbf{Z} / p) \simeq E\left[u_{1}, \ldots, u_{n}\right] \otimes P\left[\beta u_{1}, \ldots, \beta u_{n}\right]
$$

it is tempting to form the orbit space $K(\bar{V}, 1) / G$, whose $\mathrm{Z} / p$ cohomology for a group $G$ of order prime to $p$ is

$$
\left(E\left[u_{1}, \ldots, u_{n}\right] \otimes P\left[\beta u_{1}, \ldots, \beta u_{n}\right]\right)^{G} .
$$

However, it is not usually the case [8, 4, Exercise 3, pp. 135-136] that such rings of invariants are again symmetric algebras. Therefore a different approach is needed to construct $Y(V ; G)$. The crucial observation is to use an idea of Quillen [7, $\$ 1]$, and the construction of certain self-maps of $X(V ; G)$ explained in [13].

Notations and Definitions. Let $G<\mathrm{GL}\left(n ; \mathbf{Z}_{p}\right)$ be a finite subgroup. Set

$$
V:=\mathbf{Z}_{p} \oplus \stackrel{-n \rightarrow}{\cdots} \oplus \mathbf{Z}_{p}
$$

regarded as a $G$-module and let $X(V ; G)$ be the space constructed by Clark and Ewing [6]; that is, let $G$ act freely on $K(V, 2)$ via the representation and set $X(V ; G)=K(V, 2) / G$.

If $k \in \mathbf{Z} / p$ is a $p$-adic integer, let

$$
\lambda_{k}: V \rightarrow V \mid \lambda_{k}(v)=k v,
$$

so $\lambda_{k}$ is just left multiplication $k$. By [8] $\lambda_{k}$ induces a unique homotopy class of maps

$$
\Psi^{k}:=X\left(\lambda_{k}\right): X(V ; G) \rightarrow X(V ; G) .
$$


The induced map $\left(\Psi^{k}\right)^{*}: H^{2 d}(K(V, 2) ; \mathrm{Z} / p)$ is easily seen to be multiplication by $k^{d}$. Therefore by restricting to

$$
H^{*}(X(V ; G) ; \mathbf{Z} / p) \simeq H^{*}(K(V, 2) ; \mathbf{Z} / p)^{G}
$$

we see that

$$
\left(\Psi^{k}\right)^{*}\left(\rho_{i}\right)=k^{d_{i}} \rho_{i}, \quad 2 d_{i}=\operatorname{deg} \rho_{i}, \quad i=1, \ldots, n .
$$

Define the space $Y_{k}(V, G)$ by the cartesian square

$$
\begin{array}{ccc}
Y_{k}(V ; G) & \rightarrow & X(V, G)_{\hat{p}} \\
\downarrow & & \downarrow \Delta \\
X(V ; G)_{\hat{p}} & \overrightarrow{\left.\vec{q}^{k}\right)} & X(V, G)_{\hat{p}} \times X(V, G)_{\hat{p}}
\end{array}
$$

where the subscript $\hat{p}$ denotes the Bousfield-Kan [5] functorial $p$-adic completion, and $\Delta$ is the diagonal map. Our immediate goal is to compute $H^{*}(Y(V, G) ; \mathrm{Z} / p)$ under the usual assumptions, viz, that $G$ is generated by pseudo reflections and $p \nmid|G|$. The reason for passing to $p$-adic completions is simply this. Note that $\pi_{1}(X(V, G))=G$ is by hypothesis a finite group of order prime to $p$. Therefore by [5, VIII. 3.2] $X(V, G)_{\hat{p}}$ is simply connected and the natural map

$$
H^{*}\left(X(V, G)_{\hat{p}} ; \mathbf{Z} / p\right) \rightarrow H^{*}(X(V, G) ; \mathbf{Z} / p)
$$

is an isomorphism. Therefore

$$
H^{*}\left(X(V ; G)_{\hat{p}} ; \mathbf{Z} / p\right) \simeq P\left[\rho_{1}, \ldots, \rho_{n}\right] \simeq P(\bar{V})^{G}
$$

where $\bar{V}=\mathbf{Z} / p \otimes_{\mathbf{z}_{p}} V$ and $\prod_{i=1}^{n} \operatorname{deg} \rho_{i}=2^{n}|G|$.

Consider the Eilenberg-Moore spectral sequence of the fibre square (Y) [9]. This gives

$$
\begin{aligned}
& E_{r} \Rightarrow H^{*}(Y(V, G) ; \mathbf{Z} / p), \\
& E_{2}=\operatorname{Tor}_{P[\rho] \otimes P[\rho]}(P[\rho], P[\rho]),
\end{aligned}
$$

where $\rho:=\left(\rho_{1}, \ldots, \rho_{n}\right)$. The action of $P[\rho] \otimes P[\rho]$ on the right-hand variable is via the multiplication $\mu: P[\rho] \otimes P[\rho] \rightarrow P[\rho]$. The kernel of $\mu$ is the Borel ideal

$$
\left(\rho_{1} \otimes 1-1 \otimes \rho_{1}, \ldots, \rho_{n} \otimes 1-1 \otimes \rho_{n}\right)
$$

and so some change of rings arguments (see [9, Part I, 3 or 10] for this sort of argument) imply

$$
E_{2} \simeq H\left\{P\left[\rho_{1}, \ldots, \rho_{n}\right] \otimes E\left[\sigma_{1}, \ldots, \sigma_{n}\right] ; d\right\}
$$

where

$$
d \sigma_{i}=\left(1-k^{d_{i}}\right) \rho_{i}, \quad 2 d_{i}=\operatorname{deg} \rho_{i}, \quad i=1, \ldots, n .
$$

Set $r_{i}=\nu_{p}\left(1-k^{d_{i}}\right)$, so that $1-k^{d_{i}}=p^{r_{i}} q_{i}$ where $\left(q_{i}, p\right)=1$. One has

PROPOSITION 1. With the notations preceding

$$
H^{*}\left(Y_{k}(V ; G) ; \mathbf{Z} / p\right) \simeq \frac{E\left[\sigma_{1}, \ldots, \sigma_{n}\right] \otimes P\left[\rho_{1}, \ldots, \rho_{n}\right]}{\left(\sigma_{i}, \rho_{l} \mid r_{i}=0\right)}
$$

and $\beta_{r_{i}}\left(\sigma_{l}\right)=\rho_{l}, r_{i} \neq 0$. 
Proof. The preceding discussion shows

$$
E_{2} \simeq E\left[\sigma_{1}, \ldots, \sigma_{n}\right] \otimes P\left[\rho_{1}, \ldots, \rho_{n}\right] /\left(\sigma_{i}, \rho_{i} \mid r_{1}=0\right) .
$$

where the bigradings are given by

$$
\text { bideg } \rho_{i}=\left(0,2 d_{1}\right), \quad \text { bideg } \sigma_{i}=\left(-1,2 d_{i}\right), \quad i=1 \ldots, n .
$$

The usual argument [10] then shows that $E_{2}=E_{\infty}$ with no extension problem. The behavior of the Bocksteins can be determined as in [7, \$3, Lemma 5].

Of course, choosing $k=p+1$ we see that $\nu_{p}\left(1-(p+1)^{d}\right)=1$ for any integer $d$ relatively prime to $p$. Thus we have

COROLlaRy 2. With the notations preceding we have

$$
H\left(Y_{k_{p}}(V ; G) ; \mathbf{Z} / p\right) \simeq E\left[\sigma_{1}, \ldots, \sigma_{n}\right] \otimes P\left[\rho_{1}, \ldots, \rho_{n}\right]
$$

where $P\left[\rho_{1}, \ldots, \rho_{n}\right] \simeq P(V)^{G}$ and

$$
\beta \sigma_{i}=\rho_{i}, \quad i=1, \ldots, n \text {. }
$$

To see that we have constructed enough spaces to prove the theorem we note

LeMmA 3. Suppose $p$ an odd prime, and

$$
S^{*}:=E\left[\tau_{1}, \ldots, \tau_{n}\right] \otimes P\left[\rho_{1}, \ldots, \rho_{n}\right]
$$

is an unstable algebra over the Steenrod algebra. Set

$$
P^{*}:=S^{*} /\left(\tau_{1}, \ldots, \tau_{n}\right) \simeq P\left[\rho_{1}, \ldots, \rho_{n}\right] .
$$

Then $P^{*}$ is an unstable algebra over the algebra of reduced powers.

Proof. Clearly it suffices to show that the ideal $\left(\tau_{1}, \ldots, \tau_{n}\right)$ is closed under the action of the algebra of reduced powers. By the Cartan formula it is sufficient to check $P^{j} \tau_{i} \in\left(\tau_{1}, \ldots, \tau_{n}\right), 1 \leqslant i \leqslant n$, for all $j$. But $P^{j}$ is of even degree and $\tau_{i}$ is of odd degree. Therefore $P^{j} \tau_{i}$ must be a linear combination of monomials, each one of which is divisible by at least one $\tau_{k}$, or $P^{j} \tau_{i}=0$. In any case $P^{j} \tau_{i} \in\left(\tau_{1}, \ldots, \tau_{n}\right)$ as required.

Proof of TheOREM. By Lemma $3 P\left[\rho_{1}, \ldots, \rho_{n}\right]$ is an unstable algebra over the algebra of reduced powers. So by [1] there is a finite $p$-adic hyperplane group $G<\mathrm{GL}\left(n ; \mathbf{Z}_{p}\right)$ of order prime to $p$ such that $P\left[\rho_{1}, \ldots, \rho_{n}\right]=P(\bar{V})^{G}$ where $\bar{V}:=\mathbf{Z} / p \otimes_{\mathbf{Z}_{p}}\left(\mathbf{Z}_{p}\right)^{n}$. Corollary 2 now completes the proof.

REMARKS. By utilizing maps $X(f): X\left(V^{\prime}, G^{\prime}\right) \rightarrow X\left(V^{\prime \prime}, G^{\prime \prime}\right)$ induced by morphism $[13,12] f:\left(V^{\prime}, G^{\prime}\right) \rightarrow\left(V^{\prime \prime}, G^{\prime \prime}\right)$ one can construct topological spaces whose $\mathrm{Z} / p$ cohomology has the form

$$
\begin{gathered}
S^{*}:=E\left[\tau_{1}, \ldots, \tau_{n}\right] \otimes P\left[\rho_{1}, \ldots, \rho_{n}\right], \\
\operatorname{deg} \rho_{i} \neq 0(p), \quad \operatorname{deg} \tau_{i} Z-1 \bmod p
\end{gathered}
$$

when $\beta \tau_{1}, \ldots, \beta \tau_{n} \in P\left[\rho_{1}, \ldots, \rho_{n}\right]$ are an ESP sequence generating a subalgebra closed under the $\mathcal{P}^{*}$ action. 


\section{REFERENCES}

I. J. F. Adams and C. W. Wilkerson, Finite $H$-spaces and algebras over the Steenrod algebra, Ann. of Math. (2) 111 (1980), 95-143.

2. J. Aguade, Cohomologv algebras with two generation, Math. Z. 177 (1981), 289-294.

3. P. F. Baum and L. Smith, The real cohomology of differential fiher hundles, Comment. Math. Helv. 42 (1967), 171-179.

4. N. Bourbaki, Groupes et algèbres de Lie, Chapter V, Hermann, Paris, 1962.

5. A. K. Bousfield and D. M. Kan, Homotopy limits, completions and localizations, Lecture Notes in Math., vol. 304, Springer-Verlag, Berlin and New York, 1972.

6. A. Clark and J. Ewing. The realization of polynomial algebras as cohomology rings, Pacific J. Math. 50 (1974), 425-434.

7. D. Quillen, On the cohomology and $K$ theory of general linear groups over finite fields, Ann. of Math. (2) 96 (1972), 552-586.

8. L. Solomon, Invariants of finite reflection groups, Nagoya Math. J. 22 (1963), 57-64.

9. L. Smith, Homological algebra and the Eilenberg-Moore spectral sequence. Trans. Amer. Math. Soc. 129 (1967), 58-93.

10. Cohomologv of stable two stage Postnikov systems. Illinois J. Math. 11 (1967), 310-329

11. On the rational cohomologv of the free loop space. Amer. J. Math. 103 (1981), 887-910.

12. A note on the realization of graded intersection algebras as the cohomologv of a space. Quart.

J. Math. Oxford Ser. (2) 83 (1982), 379-384.

13. The homotopy classification of maps between certain spaces with polynomial cohomologi: (jöttingen Univ. Preprint SS-1981.

14. L. Smith and R. M. Switzer, Polynomial algehras over the Steenrod algehra, Variations on a theme of Adams and Wilkerson (to appear).

Institut des Hautes Études Scientifiques, 91440-Bures-sur-Yvette. France

Current address: Mathematisches Institut. Bunsenstrasse 3/5. D-3400 Göttingen. Federal Republic of (iermany 